\title{
Desarrollo de modelos NIRS de predicción para el análisis de la finura de fibras textiles de vicuña y llama
}

\section{Development of NIRS prediction models for fineness analysis of vicuna and llama textile fibers}

Presentación: 25/10/2019

Doctorando:

\section{José Ignacio Amorena}

CONICET-INTA. Estación Experimental Agropecuaria Catamarca, Ruta Provincial 33, Km 4,5, Sumalao, Valle Viejo, Catamarca, Argentina. E-Mail: amorena.jose@inta.gob.ar

Director/es:

\section{Fernández de Ahumada, Elvira (Directora)}

Departamento de Matemáticas, Universidad de Córdoba (UCO). Córdoba, España

\section{Álvarez, Dolores María Eugenia (Co-directora)}

Centro de Investigación y Tecnología Química (CITeQ), Facultad Regional Córdoba, Universidad Tecnológica Nacional (FRC-UTN). Córdoba, Argentina.

\section{Rigalt, Francisco (Co-director/tutor)}

Instituto Nacional de Tecnología Agropecuaria, Estación Experimental Agropecuaria Catamarca, Ruta Provincial 33, Km 4,5, Sumalao, Valle Viejo, Catamarca, Argentina.

\section{Resumen}

La producción de fibras textiles de llama y vicuña es una de las actividades de mayor valor ambiental, cultural y económico para la región alto-andina de Catamarca. Por esto es necesario desarrollar una tecnología que permita analizar de manera rápida y sustentable los parámetros de calidad de este material. En este trabajo se evaluaron, a través de los estadísticos R², SECV, SEV y RPD, distintos tratamientos y modelos NIRS de predicción para determinar la Finura de muestras de llama y vicuña. Los resultados mostraron que los mejores modelos se obtuvieron con ninguno o con el mínimo tratamiento. Los valores de $\mathrm{R}^{2}<0.7$ y $\mathrm{RPD} \cong 1.7$ obtenidos, dieron cuenta de la necesidad de aumentar el tamaño muestral y de explorar en el desarrollo de técnicas alternativas de regresión.

Palabras claves: NIRS, Fibras textiles, Llama, Vicuña.

\begin{abstract}
The production of llama and vicuna textile fibres is one of the more valuable environmental, cultural and economic activity in the High-Andean region of Catamarca. It is necessary to develop a technology that allows to analyze this material quickly and sustainably. In this work, different treatments and NIRS prediction models were evaluated through R2, SECV, SEV and RPD statistics to predict Fineness in samples of llama and vicuna. The results showed that

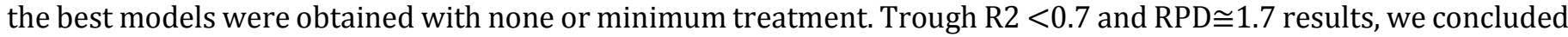
that it is necessary to increase the sample size and explore the development of alternative regression techniques.
\end{abstract}

Keywords: NIRS, Textile fibers, Llama, Vicuna.

\section{Introducción}

Los camélidos sudamericanos domésticos (llama, Lama glama y alpaca, Vicugna pacos) y silvestres (guanaco, Lama guanicoe y vicuña, Vicugna vicugna) son recursos genéticos de alto valor socioeconómico. Adaptados a la región altoandina de Argentina, Perú, Bolivia, Chile y Ecuador (E. Quispe \& Mueller, 2009), estos animales son los principales proveedores de carne, cueros y fibra (Echenique, Echenique, Fava, Alcoba, \& Quiroga Mendiola, 2015; Iñiguez, Alem, Wauer, \& Mueller, 1998). En Argentina, las fibras especiales de llama y vicuña presentan una dicotomía en cuanto a su 
comercialización; mientras que la primera está desvalorizada en el mercado nacional e internacional (2 a 4 us $\$ / \mathrm{kg}$ )(PROLANA, 2019), un vellón sucio de vicuña puede alcanzar valores de hasta 500 us $\$ / \mathrm{kg}$ (Rigalt, 2011). Atentos a esto, los productores de fibras especiales deben ser capaces de responder a las oportunidades que ofrece la creciente demanda de tejidos naturales y la preocupación por producciones sustentables ambiental, cultural y económicamente. Para poder dar respuesta a estas necesidades resulta clave disponer de un sistema analítico capaz de proporcionar de manera rápida y confiable la información necesaria sobre aquellos parámetros relacionados con la calidad de las fibras.

La espectroscopía de infrarrojo cercano (NIRS, por sus siglas en inglés, Near Infra Red Spectroscopy) se ha convertido en las últimas décadas en uno de los métodos de detección más utilizados y prometedores en el sector agroalimentario. Esto es porque ofrece un análisis rápido, versátil, de bajo costo, con nula o mínima preparación de la muestra, sin utilización de reactivos ni producción de residuos y la posibilidad de ser implementada in situ. Su funcionamiento se basa en la interacción de la luz en la región del infrarrojo cercano (780-2500 nm) con la materia de la muestra a analizar. Como consecuencia de dicha interacción se obtiene un espectro de absorción que se correlaciona con parámetros de composición química, así como con otros físicos o atributos cualitativos (Roggo et al., 2007).

Las fibras textiles de origen animal presentan un perfil complejo de analizar, ya que se trata de un material altamente heterogéneo, no solamente por su composición intrínseca (Hillbrick, 2012), sino porque en una pequeña cantidad de fibra se pueden encontrar restos vegetales, tierra y otros residuos que dificultan los procesos de medición (E. C. Quispe, Chipa, \& Pinares, 2015). El principal parámetro para determinar la calidad de la fibra es la Finura Media FM, cuantificada en micras $(\mu \mathrm{m})$ (Sommerville, 2007). Pero además existen otros parámetros que complementan este valor: el Desvío Estándar de la finura (DS) , Factor de Confort (FC), Coeficiente de Variación (CV), la Curvatura de Ondulación, color, tipo de mecha, etc. (Botha \& Hunter, 2010).

En muchos trabajos se ha evaluado la capacidad de la tecnología NIRS para predecir las características físicas de las fibras textiles. Sin embargo, los datos obtenidos dan cuenta de la necesidad de perfeccionar los métodos de obtención, análisis y procesamiento de las señales espectrales, con el fin de mejorar la precisión de los resultados (Alarcón Buhofer, 2012; Gishen \& Cozzolino, 2007; Slack-Smith, Fong, \& Douglas, 2008; Wu \& He, 2008).

El presente trabajo se enmarca dentro de la tesis doctoral "Desarrollo de metodología NIRS para la evaluación cuantitativa y cualitativa de fibras especiales de llama y vicuña", cuyo objetivo general es evaluar el potencial de esta tecnología para el diagnóstico de calidad de fibras especiales, hilados y confecciones artesanales e industriales, como así también para el aporte al diseño de políticas públicas y desarrollo de sistemas de mercados y precios.

El objetivo específico de este trabajo es el desarrollo y validación de modelos de predicción NIRS para la cuantificación de la Finura Media, parámetro de mayor interés en relación a la calidad de las fibras de vicuña y llama.

\section{Materiales y Métodos}

Se analizaron 121 vellones de vicuña y 169 de llama, tomados de la zona del costillar medio del animal (PROLANA, 2010). Las muestras fueron subdivididas para su análisis convencional, usando el instrumento de medición OFDA 2000 (IWTO 47-2013, 2013; Sommerville, 2007) del Laboratorio de Fibras Textiles en la Estación Experimental del Instituto Nacional de Tecnología Agropecuaria de Bariloche. Los parámetros analizados fueron FM $(\mu \mathrm{m})$, DS $(\mu \mathrm{m})$; CV (\%) y FC (\%), entendido como el porcentaje de fibras mayores a $30 \mu \mathrm{m}$.

Las muestras fueron escaneadas con un espectrómetro FOSS NIRS DS 2500 en la región espectral Visible e Infrarrojo (400 a $2500 \mathrm{~nm}$ ), en una cápsula portamuestras circular de $12 \mathrm{~cm}$ de diámetro x $4 \mathrm{~cm}$ de alto. Cada espectro fue recogido a intervalos de $0.5 \mathrm{~nm}$, totalizando 4200 datos.

Para el tratamiento matemático de los espectros y el desarrollo de los modelos de predicción se utilizó el programa computacional de desarrollo de calibraciones WinISI 4.

Las muestras de vicuña fueron escaneadas sin peinar, mientras que las de llama se escanearon inicialmente sin peinar y luego fueron peinadas con un cepillo de carda para ser escaneadas posteriormente (Amorena, Álvarez, Rigalt, \& Fernández de Ahumada, 2018) (tabla 1).

Tabla 1. Clasificación de los grupos de modelos de calibración.

\begin{tabular}{lllll}
\hline Grupo & $\begin{array}{l}\mathrm{N}^{\circ} \text { de } \\
\text { muestras }\end{array}$ & $\begin{array}{l}\mathrm{N}^{\circ} \text { de } \\
\text { calibraciones }\end{array}$ & Vellón & Tratamiento \\
\hline 1 & 121 & 48 & Vicuña & Sin peinar \\
2 & 169 & 48 & Llama & Sin peinar \\
3 & 169 & 48 & Llama & Peinada \\
\hline
\end{tabular}


El total de espectros de cada especie se ordenó de forma ascendente según la distancia al centro de la población, siendo estas distancias medidas en base a la $\mathrm{H}$ de Mahalanobis (Moron \& Cozzolino, 2003). Una vez ordenadas, se tomó 1 de cada 3 para constituir el colectivo de calibración, y el resto se utilizó como colectivo de validación, con el que se evaluó el error de los modelos predictivos desarrollados (Williams, 2001).

\section{Resultados y Discusión}

El desarrollo de los modelos de predicción consistió en una combinación entre distintos pretratamientos matemáticos de la señal espectral y tres rangos del espectro: el rango completo VIS-NIR (4200 datos); la fracción NIR de 1100 a 2500nm (2800 datos); y el rango de longitudes de onda que comprenden los armónicos y combinaciones de los enlaces $\mathrm{N}-\mathrm{H}$, asociados a las proteínas (680 datos).

Los pretratamientos matemáticos se utilizaron para eliminar o corregir el posible ruido espectral o scattering, generado por el efecto de dispersión que genera la luz al incidir en el material de la muestra (Wetzel, 1983).

Por un lado, los pretratamientos derivativos que se utilizaron fueron: 0-0-1-1; 1-5-3-1 y 2-5-3-1. Donde el primer dígito indica el orden de la derivada; el segundo el número de datos que incluirá la ecuación de derivación; y el tercer y cuarto dígito indican el número de datos que se utilizarán para el suavizado de la curva.

Previo a la aplicación de los pretratamientos multiplicativos o de corrección de scattering, se probaron modelos sin ningún tipo de tratamiento. Posteriormente, se aplicaron los tratamientos Standard Normal Variate \& Detrend (SNVD), y Weighted Multiplicative Scatter Correction (WMSC)(Fearn, Riccioli, Garrido-Varo, \& Guerrero-Ginel, 2009). En la imagen 1 , se observan los espectros obtenidos de cada combinación de pre-tratamientos.
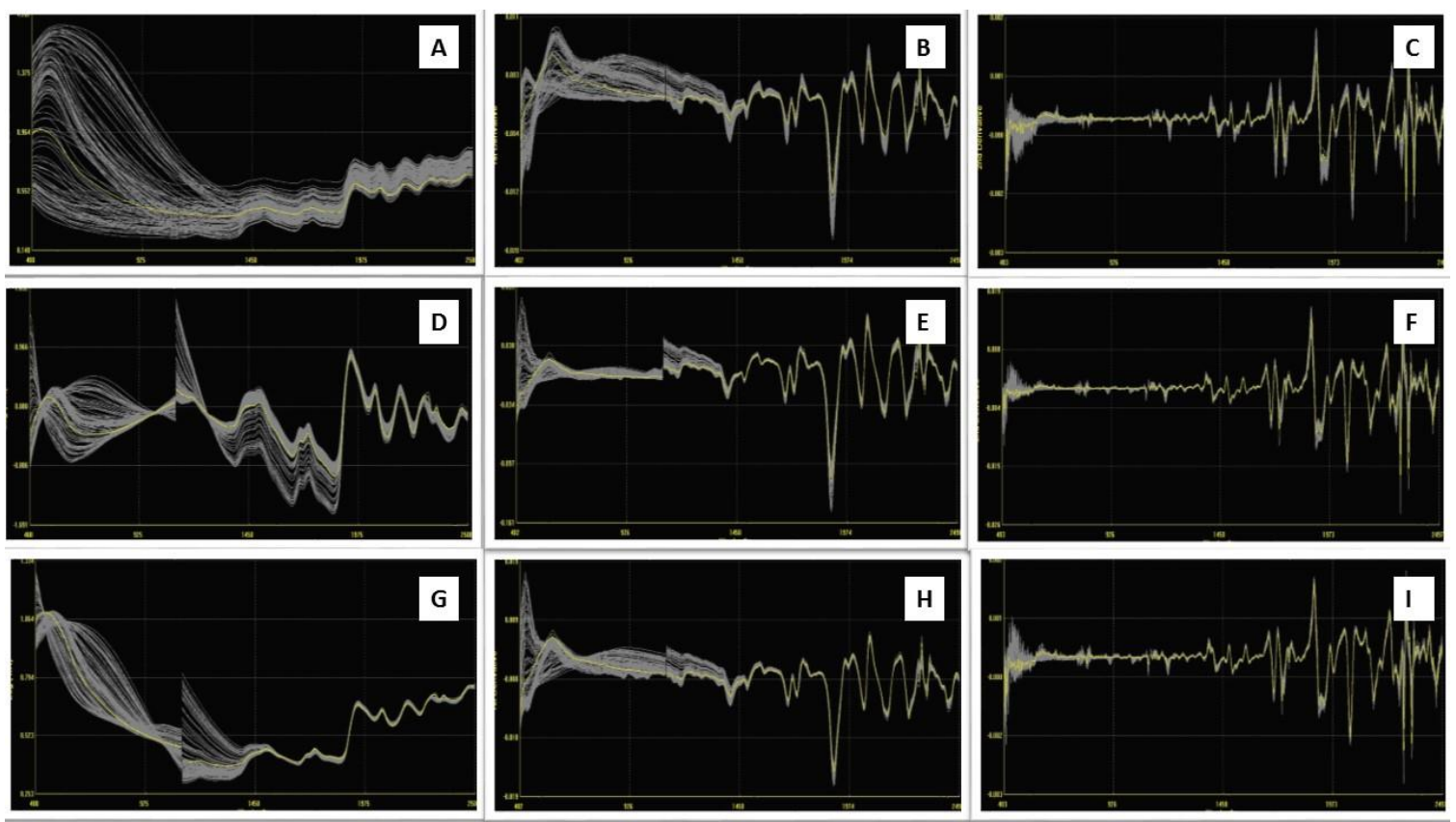

Imagen 1. Espectros de llama en la región VIS-NIR con diferentes pretratamientos matemáticos. Absorbancia (log 1/R) en función de longitud de onda $(400-2500 \mathrm{~nm})$. A) sin derivada ni corrección de scatter. B) $1^{\circ}$ derivada sin corrección de scatter, C) $2^{\circ}$ derivada sin corrección de scatter. D) sin derivada con Standard Normal Variate \& Detrend. E) $1^{\circ}$ derivada con standard normal variate $\&$ detrend. F) $2^{\circ}$ derivada con standard normal variate $\&$ detrend. G) sin derivada con weighted multiplicative scatter correction. H) $1^{\circ}$ derivada con weighted multiplicative scatter correction. I) $2^{\circ}$ derivada con weighted multiplicative scatter correction.

Para el desarrollo de las calibraciones se utilizó el método de regresión conocido como Modified-Partial Least Squares (M-PLS). En todos los casos, se utilizó la técnica Leave-one-out para llevar a cabo la validación cruzada.

Una vez constituido el colectivo de muestras con sus espectros y obtenidos sus correspondientes parámetros de calidad por métodos de laboratorio convencionales, se procedió al modelado de las calibraciones.

La capacidad predictiva de cada modelo se evaluó en base a los estadísticos coeficiente de determinación ( $\left.\mathrm{R}^{2}\right)$; los errores de validación cruzada (SECV, por sus siglas en inglés Standard Error of Cross Validation), y de validación externa (SEV, por sus siglas en inglés, Standard Error of Validation), que es el error que obtiene el modelo al predecir el archivo de validación; y el valor predictivo residual (RPD, por sus siglas en inglés Residual Predictive Value), que corresponde al ratio entre el desvío estándar del laboratorio y el error estándar de la validación cruzada (Fearn, 2002). El parámetro RPD es un valor adimensional, e indica la capacidad del modelo para ser empleado con fines analíticos (Gishen \& Cozzolino, 2007). 


\section{Vicuña}

Se realizaron 48 calibraciones combinando los distintos pretratamientos derivativos y multiplicativos entre sí y los diferentes rangos espectrales. En la tabla 2 se muestran los modelos con la mejor capacidad predictiva de acuerdo a la combinación entre los estadísticos R², SECV, SEV y RPD.

Como se puede observar, los mayores valores de $\mathrm{R}^{2}$ se evidencian en los casos en donde no se aplicaron pretratamientos (0-0-1-1 none). En cuanto al rango espectral, no se manifestó diferencia entre valores obtenidos mediante el empleo del espectro completo (4200 datos) y el rango del infrarrojo (2800 datos).

Tabla 2. Modelos de calibración del Grupo 1 (vicuña sin peinar) para Finura.

\begin{tabular}{llllllllll}
\hline Parámetro & Modelo & $\mathrm{n}$ cal & $\mathrm{n}$ val & $\mathrm{N}^{\circ}$ datos & Pre-tratamientos & $\mathrm{R}^{2}$ & $\begin{array}{l}\text { SECV } \\
(\mu \mathrm{m})\end{array}$ & $\begin{array}{l}\text { SEV } \\
(\mu \mathrm{m})\end{array}$ & $\mathrm{RPD}$ \\
\hline \multirow{4}{*}{ Finura } & 1 & 91 & 30 & 4200 & $0-0-1-1$ none & 0,61 & 0,731 & 0,621 & 1.71 \\
& 2 & 81 & 40 & 4200 & $0-0-1-1$ none & 0,57 & 0,732 & 1,033 & 1.71 \\
& 3 & 81 & 40 & 2800 & $0-0-1-1$ none & 0,56 & 0,727 & 1,080 & 1.72 \\
& 4 & 91 & 30 & 2800 & $0-0-1-1$ none & 0,54 & 0,818 & 0,744 & 1.53 \\
& 5 & 91 & 30 & 4200 & $0-0-1-1$ snvd & 0,53 & 0,755 & 1,107 & 1.66 \\
\hline
\end{tabular}

n cal: muestras usadas para el modelo de calibración, $\mathrm{n}$ val: muestras usadas para la validación externa, $\mathrm{R}^{2:}$ coeficiente de determinación, SECV: error estándar de la validación cruzada, SEV: error estándar de la validación externa, RPD: valor predictivo residual:

\section{Llama}

Se realizaron 48 calibraciones considerando los grupos 2 y 3 y combinando los distintos pretratamientos derivativos y multiplicativos entre sí y los diferentes rangos espectrales. En las tablas 3 y 4 se observan los resultados obtenidos. Debido a que se trata del mismo material, es posible comparar los parámetros entre ambos grupos.

Tabla 3. Modelos de calibración del Grupo 2 (llama sin peinar) para Finura.

\begin{tabular}{llllllllll}
\hline Parámetro & Modelo & $\mathrm{n}$ cal & $\mathrm{n}$ val & $\mathrm{N}^{\circ}$ datos & Pre-tratamientos & $\mathrm{R}^{2}$ & $\mathrm{SECV}$ & $\mathrm{SEV}$ & $\mathrm{RPD}$ \\
\hline \multirow{4}{*}{ Finura } & 1 & 112 & 57 & 2800 & $1-5-3-1$ none & 0,70 & 2,194 & 3,128 & 1,71 \\
& 2 & 112 & 57 & 680 & $0-0-1-1$ none & 0,68 & 2,225 & 3,290 & 1,69 \\
& 3 & 112 & 57 & 2800 & $0-0-1-1$ none & 0,67 & 2,223 & 2,955 & 1,69 \\
& 4 & 112 & 57 & 4200 & $1-5-3-1$ none & 0,65 & 2,396 & 3,359 & 1,57 \\
& 5 & 112 & 57 & 680 & $1-5-3-1$ none & 0,64 & 2,169 & 3,115 & 1,73 \\
\hline
\end{tabular}

n cal: muestras usadas para el modelo de calibración, $\mathrm{n}$ val: muestras usadas para la validación externa, $\mathrm{R}^{2:}$ coeficiente de determinación, SECV: error estándar de la validación cruzada, SEV: error estándar de la validación externa, RPD: valor predictivo residual.

Tabla 4. Modelos de calibración del Grupo 3 (Ilama peinada) para Finura.

\begin{tabular}{llllllllll}
\hline Parámetro & Modelo & $\mathrm{n}$ cal & $\mathrm{n}$ val & $\mathrm{N}^{\circ}$ datos & Pre-tratamientos & $\mathrm{R}^{2}$ & $\mathrm{SECV}$ & $\mathrm{SEV}$ & $\mathrm{RPD}$ \\
\hline \multirow{4}{*}{ Finura } & 1 & 127 & 42 & 2800 & $1-5-3-1$ none & 0,70 & 2,113 & 2,175 & 1,78 \\
& 2 & 127 & 42 & 4200 & $1-5-3-1$ none & 0,68 & 2,118 & 2,051 & 1,77 \\
& 3 & 127 & 42 & 680 & $1-5-3-1$ none & 0,67 & 2,057 & 2,500 & 1,83 \\
& 4 & 112 & 57 & 2800 & $1-5-3-1$ none & 0,67 & 2,175 & 3,295 & 1,73 \\
& 5 & 112 & 57 & 4200 & $1-5-3-1$ none & 0,66 & 2,129 & 3,385 & 1,76
\end{tabular}

n cal: muestras usadas para el modelo de calibración, $n$ val: muestras usadas para la validación externa, R2: coeficiente de determinación, SECV: error estándar de la validación cruzada, SEV: error estándar de la validación externa, RPD: valor predictivo residual. 
En cuanto al análisis de los estadísticos de calibración, se observa que los valores de los modelos 1 y 2 en el SEV del grupo 3 (SEV=2.175 $\mu \mathrm{m}$ y SEV=2.051 $\mu \mathrm{m}$, respectivamente), son menores a los del grupo 2 (SEV=3.128 $\mu \mathrm{m}$ y $\mathrm{SEV}=3.290 \mu \mathrm{m}$ ), lo cual podría indicar una mejora en el tratamiento de la fibra peinada, tal como se comprobó anteriormente en ensayos de repetibilidad (Amorena, Fernández de Ahumada, Álvarez, \& Rigalt, 2018).

Los mejores modelos se obtuvieron cuando no se aplicaron tratamientos de corrección de scattering (none), pero aplicando, en la mayoría casos, el pretratamiento con primera derivada (1-5-3-1). Por otro lado, el uso del rango espectral completo (4200 datos), o porciones de éste (2800 o 680 datos) no evidenció diferencias significativas en los valores estadísticos.

Comparativamente, los resultados del estadístico $\mathrm{R}^{2}$ de los mejores modelos de cada grupo $\left(\mathrm{R}^{2}=0.61\right.$ en el grupo 1 y $\mathrm{R}^{2}=0.70$ en los grupos 2 y 3 ) fueron menores a los obtenidos en fibra de alpaca $\left(\mathrm{R}^{2}=0.88\right)$ (Alomar, Alarcón Buhofer, \& Kusanovic, 2015; Canaza-Cayo, Alomar, \& Quispe, 2013; Gishen \& Cozzolino, 2007), y lana de oveja limpia, $\left(\mathrm{R}^{2}=\right.$ 0.90 ); pero no así en lana sucia $\left(\mathrm{R}^{2}=0.50\right.$ ) (Cozzolino et al., 2005). Por otro lado, el $\mathrm{RPD} \cong 1.7$ en los tres grupos, fue mucho menor a 3, que es el umbral mínimo que debería tener un modelo de calibración para considerarse aceptable para realizar predicciones con exactitud (Fearn, 2002; Gishen \& Cozzolino, 2007). Finalmente, los SECV del grupo 1 $(\mathrm{SECV} \cong 0.7 \mu \mathrm{m})$, y grupos 2 y $3(\mathrm{SECV} \cong 2.2 \mu \mathrm{m})$, fueron mayores a los reportados en otros trabajos en alpaca $(\mathrm{SECV}=2.62 \mu \mathrm{m})$ (Alomar, Alarcón Buhofer, \& Kusanovic, 2015; Canaza-Cayo, Alomar, \& Quispe, 2013; Gishen \&

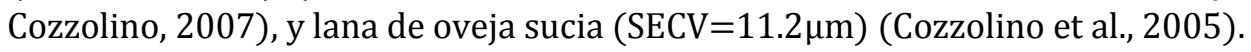

\section{Conclusiones}

El propósito del presente trabajo fue evaluar el desempeño de modelos de predicción NIRS para el parámetro Finura Media. Uno de los hallazgos más notables encontrados fue que los mejores modelos se obtuvieron con mínimo o ningún pre-tratamiento espectral. En segundo lugar, es importante destacar que no se encontraron diferencias significativas en el uso del rango espectral completo o fraccionado. Esto estaría indicando que el tratamiento de las muestras, previo a la obtención de los espectros, es fundamental para el desarrollo de los modelos de predicción.

Algunas limitaciones a destacar en este estudio fueron, por un lado, la cantidad de muestras utilizadas, ya que es necesario contar con un mayor tamaño muestral, especialmente teniendo en cuenta la heterogeneidad de la fibra, y por el otro lado, una gran limitante fue la falta de bibliografía específica, por lo que hubo que guiarse por trabajos realizados con materiales similares (fibra de alpaca y lana de oveja), lo que llevó a hacer comparaciones aproximadas y no lo suficientemente precisas.

Para poder optimizar los nuevos modelos en futuros estudios será necesario aumentar el tamaño muestral, tanto para llama como para vicuña. Además, se deberá tener en cuenta la posibilidad de explorar métodos de regresión diferentes a M-PLS, como por ejemplo regresión por redes neuronales, máquinas de vectores soporte y algoritmos locales.

\section{Agradecimientos}

Los autores agradecen el apoyo de la Dirección Provincial de Biodiversidad de la Secretaría de Estado del Ambiente y Desarrollo Sustentable de Catamarca, que facilitó el proceso de obtención de las muestras de vicuña (Resolución 320/14); a los laboratorios de Fibras Textiles y de Aceites y Grasas, de las Estaciones Experimentales del Instituto Nacional de Tecnología Agropecuaria (INTA) de Bariloche y Catamarca respectivamente; al Consejo Nacional de Investigaciones Científicas y Técnicas (CONICET); y a la Universidad Tecnológica Nacional, Facultad Regional Córdoba (UTN-FRC).

\section{Referencias}

Alarcón Buhofer, M. M. I. (2012). Predicción de la calidad de lana mediante Espectroscopía de Reflectancia en el Infrarrojo Cercano (NIRS). Universidad Austral de Chile.

Alomar, D., Alarcón Buhofer, M. M. I., \& Kusanovic, A. (2015). Predicción de la calidad de lana mediante Espectroscopía de Reflectancia en el Infrarrojo Cercano ( NIRS ). Agro Sur, 43(1), 19-24. https://doi.org/10.4206/agrosur.2015.v43n103

Amorena, J. I., Álvarez, D. M. E., Rigalt, F., \& Fernández de Ahumada, E. (2018). Desarrollo preliminar de metodología NIRS para la evaluación cuantitativa y cualitativa de fibra de vicuña (Vicugna vicugna). 3er Congreso de Ingeniería de Procesos y Productos, (1), 241-250. https://doi.org/ISBN 978-950-42-0187-8

Amorena, J. I., Fernández de Ahumada, E., Álvarez, D. M. E., \& Rigalt, F. (2018). Desarrollo preliminar de metodología NIRS para la evaluación cuantitativa y cualitativa de fibra de llama (Lama glama). 8 vo Congreso Mundial Sobre Camélidos. Oruro, Bolivia. 
Botha, A. F., \& Hunter, L. (2010). The measurement of wool fibre properties and their effect on worsted processing performance and product quality. Part 1: The objective measurement of wool fibre properties. Textile Progress, 42(4), 227-339. https://doi.org/10.1080/00405167.2010.486932

Canaza-Cayo, A. W., Alomar, D., \& Quispe, E. (2013). Prediction of alpaca fibre quality by near-infrared reflectance spectroscopy. Animal, 7(7), 1-7. https://doi.org/10.1017/S1751731113000505

Cozzolino, D., Montossi, F., \& San Julian, R. (2005). Science (VIS ) and near infrared (NIR) reflectance spectroscopy to predict fibre diameter in both clean and greasy wool samples. Animal Science, 80(3), 333-337. https://doi.org/10.1079/ASC41760333

Echenique, M., Echenique, J., Fava, R., Alcoba, D., \& Quiroga Mendiola, M. (2015). La producción y comercialización de carne de la agricultura familiar en la Puna jujeña.

Fearn, T. (2002). Assessing calibrations: SEP, RPD, RER and R2. NIR News, 13(6), 12-14. https://doi.org/https://doi.org/10.1255/nirn.689

Fearn, T., Riccioli, C., Garrido-Varo, A., \& Guerrero-Ginel, J. E. (2009). Chemometrics and Intelligent Laboratory Systems On the geometry of SNV and MSC. Chemometrics and Intelligent Laboratory Systems, 96, 22-26. https://doi.org/10.1016/j.chemolab.2008.11.006

Gishen, M., \& Cozzolino, D. (2007). Feasibility study on the potential of visible and near infrared reflectance spectroscopy to measure alpaca fibre characteristics. Animal, 1(6), 899-904. https://doi.org/10.1017/S1751731107000146

Hillbrick, L. K. (2012). Fibre Properties affecting the Softness of Wool and other Keratins. Deakin University, Australia.

Iñiguez, L. C., Alem, R., Wauer, A., \& Mueller, J. (1998). Fleece types, fiber characteristics and production system of an outstanding llama population from Southern Bolivia. Small Ruminant Research, 30(1), 57-65. https://doi.org/10.1016/s0921-4488(98)00079-0

IWTO 47-2013. (2013). In IWTO Regulations.

Moron, A., \& Cozzolino, D. (2003). Exploring the use of near infrared reflectance spectroscopy to study physical properties and microelements in soils. Journal of Near Infrared Spectroscopy, 11, 145-154.

PROLANA. (2010). Reglamento PROLANA para pequeños productores. Retrieved from https://prolana.magyp.gob.ar/

PROLANA. (2019). Informe mensual. Mercado mundial de fibras animales (Vol. 79). Retrieved from https://inta.gob.ar/sites/default/files/inta_fibras_n-39_septiembre_2019.pdf

Quispe, E. C., Chipa, L., \& Pinares, R. (2015). Análisis económico y de la producción del descerdado manual de la fibra de llamas (Lama glama) Chaku. Archivos de Zootecnia, 64(246), 191-198. https://doi.org/10.21071/az.v64i246.397

Quispe, E., \& Mueller, J. (2009). Producción de fibra de alpaca, llama, vicuña y guanaco en Sudamérica. Animal Genetic Resources Information, 45(September 2014), 1-14. https://doi.org/10.1017/S1014233909990277

Rigalt, F. (2011). Manejo de la vicuña en la República Argentina. VII Congreso de La Asociación Latinoamericana de Especialistas En Pequeños Rumiantes y Camélidos Sudamericanos, 27-32. Huancavelica, Perú.

Roggo, Y., Chalus, P., Maurer, L., Lema-Martinez, C., Edmond, A., \& Jent, N. (2007). A review of near infrared spectroscopy and chemometrics in pharmaceutical technologies. Journal of Pharmaceutical and Biomedical Analysis, 44(3 SPEC. ISS.), 683-700. https://doi.org/10.1016/j.jpba.2007.03.023

Scott, R. F., Roberts, E. M., \& Keogh, M. J. (1978). Preliminary report on the estimation of average fibre diameter of greasy wool by Near Infrared Reflectance Spectroscopy. Animal Production in Australia, 515-518.

Slack-Smith, T., Fong, D., \& Douglas, S. A. S. (2008). The Potential Application of Near-infra-red Reflectance to Estimate the Alcohol-extractable- matter Content of Scoured Wool. The Journal of The Textile Institute, 70(1), 33-33. https://doi.org/10.1080/00405007908631513

Sommerville, P. (2007). Fundamental Principles of Fibre Fineness Measurement. Kensington, Australia: Australian Wool Testing Authority Ltd.

Wetzel, D. L. (1983). Reflectance Analysis. American Chemical Society, 55(12), 1-12. https://doi.org/10.1021/ac00262a718

Williams, P. (2001). Implementation of Near Infrared technology. In Near-Infrared Technology in the Agricultural and Food Industries (2nd Editio, pp. 145-169). American Asociation of Cereal Chemists.

Wu, G., \& He, Y. (2008). Identification of fine wool and cashmere by Vis / NIR spectroscopy technology. In Liwei Zhou (Ed.), International Simposium on Photoelectronic Detection and Imaging (Vol. 6625, pp. 1-10). https://doi.org/10.1117/12.791231 